\title{
Optics for electron cyclotron resonance heating and collective Thomson scattering at the stellarator $\mathrm{W} 7-\mathrm{X}$
}

\author{
W. Kasparek ${ }^{1}$, V. Erckmann ${ }^{2}$, H.P. Laqua ${ }^{2}$, T. Stange ${ }^{2}$, M. Weißgerber ${ }^{2}$, C. Lechte ${ }^{1}$, B. Plaum ${ }^{1}$, \\ D. Moseev ${ }^{2}$, F. Leipold ${ }^{3}$, M. Petelin ${ }^{4}$, K.J. Brunner ${ }^{2}$, H. Braune ${ }^{2}$, S. Marsen ${ }^{2}$, N. Schneider ${ }^{2}$, \\ R.C. Wolf ${ }^{2}$, and the W7-X team ${ }^{2}$ \\ ${ }^{1}$ Institute of Interfacial Process Engineering and Plasma Technology, U. Stuttgart, Germany, kasparek@igvp.uni-stuttgart.de \\ ${ }^{2}$ Max-Planck Institute of Plasma Physics (IPP), Garching and Greifswald, Germany \\ ${ }^{3}$ Technical University of Denmark, Kgs. Lyngby, Denmark \\ ${ }^{4}$ Inst. of Applied Physics, Russian Academy of Science, Nizhny Novgorod, Russia
}

In December 2015, the superconducting stellarator Wendelstein-X (W7-X) [1] became operable. During the first operational phase (OP 1.1) electron cyclotron resonance heating $(\mathrm{ECRH})$ was the exclusive heating method [2], and it will remain the dominating heating system in the next operational campaign (OP 1.2). At present, 9 out of $101-\mathrm{MW}$ gyrotrons running at $140 \mathrm{GHz}$ are operable. As a versatile technique, ECRH has to drive the plasma start-up, help with wall conditioning, and provide heating and current drive, making optimum use of the spatially localized power deposition of the millimetre waves. Movable launchers allow tailoring the radial profiles of the electron temperature and the rotational transform profiles. A sophisticated control system [3] is used to manage the operation of the complete system. In addition, the system can be used for collective Thomson scattering experiments.

This paper will provide a brief review on the optical transmission system, describe its performance and first experiences, and will concentrate on new developments like in-vessel grating reflectors, Remote-steering (RS) antennas used as launchers and receivers for ECRH and collective Thomson scattering (CTS) [4], as well as possible upgrades.

\section{System overview}

Fig. 1 shows a sketch of the complete ECRH system. It consists of 10 gyrotrons with a total power close to $8 \mathrm{MW}, \mathrm{CW}$ operation. Transmission to the plasma is performed fully optically via water-cooled reflectors installed in an underground beam duct. The beams of the gyrotrons are conditioned with individual matching optics units, and transmitted in common via two multi-beam waveguides (MBWG) [5] to the experimental hall. Beam dividing optics (BDO) at the end of the MBWGs distribute the beams to 10 front-steering launchers.

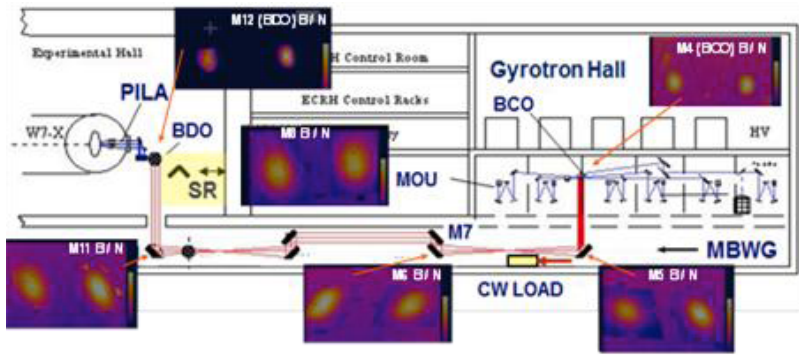

Fig. 1. Overview on the W7-X ECRH transmission system with thermal images taken on the MBWG reflectors showing beam positions relative to four markers each, as well as the beam shape on various reflectors.
In addition, two remote-steering launchers (RSL) [6] have been installed in a toroidal position where magnetic field gradient is low to study advanced current drive scenarios for example. One RSL will also be used for CTS.

\section{Transmission characteristics}

The alignment of the (in total about 150) reflectors of the transmission system and the launchers was performed by thermal imaging of the gyrotron beams on the reflector surfaces. For the MBWGs, which provide imaging from the beam combiner optics (BCO) at the input to the BDOs at the output, the alignment could be done with two beams only; all others are automatically aligned. Thermal images at various positions on the reflectors are shown in Fig. 1.

Besides ten calorimeters assigned to each gyrotron, the system employs two CW calorimetric loads installed near to the inputs of the MBWGs. By inclining one of the $\mathrm{BCO}$ reflectors, the respective beam can be directed into the load for power measurements. For measurements of the transmission efficiency, a retro-reflector SR can be inserted at the end of the MBWG (Fig. 1), and the backward travelling power can be directed into the load. Thus, by comparison of the direct and the power guided via the MBWG, a measurement of the transmission efficiency is possible, with the result shown in Fig. 2. From this and further measurements we conclude, that the efficiency of the MBWG itself is 0.972 , and the total efficiency from gyrotron to the stellarator is $>0.93$.

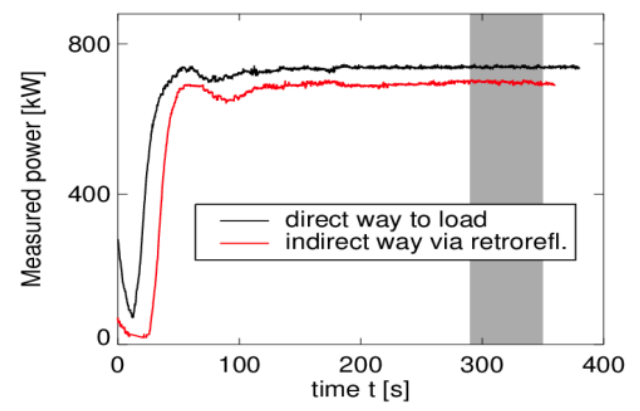

Fig. 2. Gyrotron power measured for the direct path to the load and the path via the MBWG with retro-reflectors

\section{Main launchers and in-vessel reflectors}

The ECRH power is usually injected via 10 frontsteering launchers with 2-axes movable water-cooled reflectors. They are described in [1]. The calibration of the launch directions was performed with thermal targets installed in the vessel. The typical steering range is - 
$8^{\circ}<\varphi<+35^{\circ}$ in toroidal, and $-30^{\circ}<\theta<+30^{\circ}$ in poloidal direction, giving large experimental flexibility.

For heating scenarios, with incomplete absorption of the ECRH beams (O2 and X3 heating), reflectors are installed on the inner wall opposite to the laun-chers, such that the remaining power is directed back to the plasma in a controlled way and further to areas which are insensitive to millimetre waves. Where necessary, the reflectors were designed as gratings with high efficiency in any polarisation [7], with the goal to keep the mode injected into the plasma.

\section{Remote steering launchers}

For investigations on trapped particles in $\mathrm{W} 7-\mathrm{X}$, as demonstrator for reactor-compatible ECRH launchers, as well as for CTS experiments, two ports of W7-X have been equipped with waveguide RS launchers (RSL1 and RSL5) [6]. The remote-steering properties are based on multi-mode interference in a square waveguide leading to imaging effects: For a proper length/width of the waveguide, a microwave beam at the input of the waveguide (with a defined direction set by a mirror system outside of the plasma vacuum) will exit the waveguide (near the plasma) in the same direction.

The two RS launchers are fed via switches from the main transmission system. Main features include: (i) vacuum window at the entrance, (ii) 4.6 and 5.2 long corrugated square waveguides folded by mitre bends to fit the launchers into the W7-X structure, (iii) optimized injection of the input beam for maximum angular steering of the beam launched to the plasma. The usable steering range is at least $-15^{\circ}<\varphi<+15^{\circ}$. A detailed description is given in [6].

The two launchers have been installed and aligned for operation in the next campaign (OP1.2.). High-power patterns recorded in the plasma vessel for various steering angles are shown in Fig. 3. Furthermore, RSL1 can deliver the probing beam for CTS experiments [5], measuring the ion temperature profile.

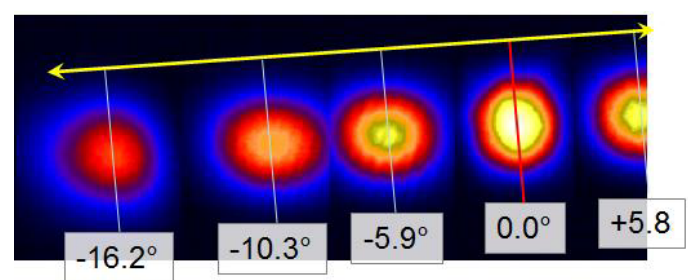

Fig. 3. High-power antenna patterns from RSL1

\section{An optical remote-steering antenna for CTS}

As receiver antenna for the CTS system, an optical remote-steering antenna was designed and built (Fig. 4). In contrast to the waveguide RSL, this antenna is broadband. Like the MBWG, it is based on the imaging properties of two confocal optics installed in series, and consists of four focusing reflectors in Z-arrangement, with surface parameter $\mathrm{p}=0.3$ [5]. Characterisation of this antenna using thermal imaging provides a beam with good quality in the angular range $-15^{\circ}<$ phi $<15^{\circ}$. Only little degradation is observed towards the limit of the steering range.
The scattered signal from this antenna is transmitted via an additional channel of the main transmission system - sharing the MWBG section with the gyrotrons - up to a spare gyrotron box, where the CTS receiver is installed.

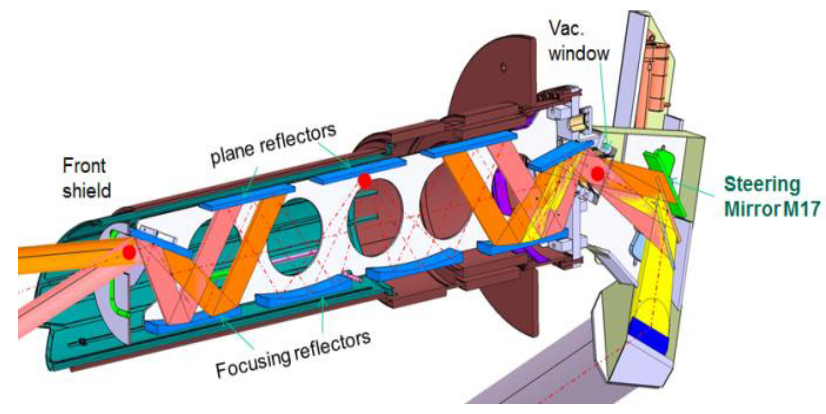

Fig. 4. Design of the optical remote-steering receiver antenna. Note the imaging of the beam pivot near the plasma to the beam pivot in the plane of the vac. window (red dots)

\section{Resonant Diplexer as switch for ECRH and spectral/spatial filter for CTS}

As a possible upgrade to ECRH and CTS, the integration of a resonant diplexer [8] is discussed. Such a device could replace the mechanical mirror switches between the main and RS launchers, and could improve the spectral purity of the gyrotron used for CTS, if necessary. A concept is based on a folded mirror resonator, which could be installed near the entrance of the MBWG or the intermediate image plane after M7 (Fig. 1).

\section{Conclusion}

The ECRH system for W7-X is characterised by high performance, and provides large experimental flexibility. The present upgrades for CTS experiments enhance the diagnostic capabilities of W7-X. An overview of past and upcoming experiments is presented in [2].

\section{References}

1. V. Erckmann, et al., Electron Cyclotron Heating for W7-X: Physics and Technology // Fusion Sci. Technol. 2007. V.52, No.2. P. 291-312.

2. J. Brunner et al., Continuous High-Power Microwave Heating at the W7-X Stellarator // this conference.

3. H. Braune et al., Enhancements of the W7-X ECRH facility with respect to the next experiment campaign OP1.2// this conference.

4. D. Moseev et al., Design and commissioning of the collective Thomson scattering diagnostic at Wendelstein 7-X // 18th Laser-aided Plasma Diagnostics, Prague, Sept. 2017.

5. L. Empacher and W. Kasparek, Analysis of a MultipleBeam Waveguide for Free-Space Transmission of Microwaves // IEEE Trans. Antennas Prop. 2001. V.49, No.3. P.483-493.

6. B. Plaum et al, Design of a remote-steering antenna for ECRH heating in the stellarator Wendelstein 7-X. // Fusion Engineering and Design 2015. V.96-97. P.568-572.

7. H. Höhnle et al., Extension of the ECRH operational space with $\mathrm{O} 2$ and X3 heating at ASDEX Upgrade // Nucl. Fusion, 2011. V.51 No.8, 083013.

8. Z. Wu et al., Design and Characterization of a $170-\mathrm{GHz}$ Resonant Diplexer for High-Power ECRH Systems. IEEE Trans. Microwave Theory Techn. 2015. V.63, No.10. P.3537-3546. 